\title{
Validação de diagnósticos de enfermagem: desafios e alternativas
}

\author{
Validation of nursing diagnosis: challenges and alternatives \\ Validación de diagnósticos de enfermería: retos y alternativas
}

\author{
Marcos Venícios de Oliveira Lopes', Viviane Martins da Silva', Thelma Leite de Araujo' \\ ' Universidade Federal do Ceará, Faculdade de Farmácia Odontologia e Enfermagem, \\ Departamento de Enfermagem. Fortaleza-CE, Brasil.
}

Submissão: 31-08-2013 Aprovação:01-09-2013

\begin{abstract}
RESUMO
Estudos sobre validação de diagnósticos de enfermagem têm sido fortemente influenciados por referenciais metodológicos desenvolvidos na década de 1980. A adequação destes modelos para validar elementos que compõem um diagnóstico de enfermagem tem sofrido várias críticas. Este artigo tem por objetivo discutir estas limitações e descrever abordagens alternativas para superar as críticas aos modelos tradicionais. Uma análise crítica baseada na literatura publicada recentemente sobre novas abordagens na pesquisa com diagnósticos foi tomada como base para as reflexões apresentadas. São descritos métodos alternativos para a análise de conceitos aplicada aos processos de validação de diagnósticos de enfermagem; métodos para a análise de conteúdo diagnóstico por juízes proficientes; e métodos de validação clínica de indicadores e fatores etiológicos. Conclui-se que existem poucos estudos com estas novas abordagens e que a complexidade das análises é um aspecto que dificulta sua implementação.
\end{abstract}

Descritores: Diagnóstico de Enfermagem; Pesquisa em Enfermagem; Medidas em Epidemiologia.

\section{ABSTRACT}

Studies on validation of nursing diagnoses have been strongly influenced by the methodological framework developed in the 1980s. The suitability of these models to validate elements that make a nursing diagnosis has undergone several criticisms. This article aims to discuss these limitations and describe alternative approaches to overcome the criticism of traditional models. A critical analysis based on recent published literature on new approaches in research diagnoses was taken as basis for the discussions presented. Alternative methods are described for the concept analysis applied the procedures for validation of nursing diagnoses; methods for diagnostic content analysis by proficient judges, and methods for clinical validation of indicators and etiological factors. Conclude that there are few studies with these new approaches and the complexity of the analysis is an aspect that hinders its implementation.

Key words: Nursing Diagnosis; Nursing Research; Epidemiologic Measurements.

\section{RESUMEN}

Estudios de validación de diagnósticos enfermeros han sido fuertemente influenciados por el marco metodológico desarrollado en los años 1980. La adecuación de estos modelos para validar los elementos componentes de un diagnóstico enfermero ha sido objeto de numerosas críticas. Este artículo tiene como objetivo discutir estas limitaciones y describir los enfoques alternativos para superar las críticas los modelos tradicionales. Un análisis crítico basado en la literatura reciente publicada sobre los nuevos enfoques de la investigación con diagnósticos se tomó como base para las reflexiones presentadas. Otros métodos se describen para el análisis de los conceptos aplicados a los procesos de validación de los diagnósticos enfermeros, los métodos de análisis de contenido diagnóstico por jueces competentes, y métodos de validación clínica de indicadores y factores etiológicos. Se concluye que hay pocos estudios con estos nuevos enfoques y la complejidad del análisis es un aspecto que dificulta su aplicación.

Palabras clave: Diagnóstico de Enfermería; Investigación en Enfermería; Mediciones Epidemiológicas. 


\section{INTRODUÇÃO}

A pesquisa sobre diagnósticos de enfermagem tem sido fortemente influenciada pela proposta de Fehring ${ }^{(1)}$. Um levantamento desenvolvido sobre os modelos de validação de diagnósticos de enfermagem utilizados nas produções dos programas de pós-graduação do Brasil mostrou que 58,3\% dos estudos utilizavam as recomendações deste autor ${ }^{(2)}$. Fehring propôs três modelos principais: Validação de conteúdo diagnóstico, Validação clínica e Validação de diagnóstico diferencial(3). Estas etapas, aliadas a uma etapa prévia de análise de conceitos, têm formado a base dos estudos de validação de diagnósticos de enfermagem.

Apesar das características positivas destes métodos, pesquisadores têm relatado algumas dificuldades e limitações dos mesmos, tanto na analise do conceito como na identificação e formação do grupo de experts.

$\mathrm{Na}$ realização da etapa de análise de conceito, alguns estudos têm encontrado dificuldade para obter material adequado em qualidade e quantidade para a construção ou mesmo revisão de diagnósticos de enfermagem. Um estudo sobre o risco para Débito cardíaco diminuído descreveu que uma das limitações enfrentadas foi um número insuficiente de artigos para uma revisão consistente do diagnóstico ${ }^{(4)}$. Ainda com referência a esta etapa, discute-se o fato de que os modelos tradicionais de análise de conceito não foram especificamente desenvolvidos para processos de validação de diagnósticos de enfermagem ${ }^{(5)}$. Com isso, o uso destes modelos esbarra na falta de direcionamento sobre como os elementos de um diagnóstico (indicadores clínicos e fatores etiológicos) devem ser tratados nesta análise e extraídos do material selecionado.

O processo de validação de conteúdo recomendado por Fehring(3) inclui a identificação de experts para compor um painel com a finalidade de analisar os componentes e suas definições supostamente desenvolvidas com a análise de conceito. Novamente os estudiosos têm descrito dificuldades para o desenvolvimento desta etapa, sobretudo em relação ao processo de captação de experts ${ }^{(6)}$. Os critérios de seleção propostos pelo autor parecem privilegiar a formação acadêmica em detrimento da experiência clínica. Isto aparentemente, vai de encontro às recomendações internacionais para o estabelecimento de expertise em enfermagem ${ }^{(7-8)}$. Uma possível justificativa para isto seria o fato de que Fehring propôs seu método no final da década de 1980, um período em que a titulação acadêmica era obtida por pesquisadores experientes e que, de certa forma, aliavam duas características importantes, conhecimento teórico e experiência clínica, o que não obrigatoriamente se aplica ao momento atual.

A partir destas situações observou-se que alguns pesquisadores passaram a fazer adaptações dos critérios de seleção propostos por Fehring ${ }^{(2)}$. Estas recomendações incluíam a adoção de um tempo de experiência prática, mudança nas pontuações inicialmente propostas, acréscimo de outras características dos supostos experts que indicariam maior aproximação com o tema ou mesmo a especificação de conhecimento ou experiência com um diagnóstico específico.
Apesar dos esforços, estas adaptações para uma seleção mais adequada parecem que não surtem o efeito desejado. Um estudo sobre risco de aspiração em pacientes com acidente vascular encefálico ${ }^{(9)}$ descreveu grande dificuldade na obtenção de um número mínimo de experts, ao utilizar oito critérios adaptados, dois dos quais eram obrigatórios, e buscar aliar a formação acadêmica com a experiência clínica na temática em estudo. Ainda com referência à composição do grupo de experts, há controvérsias sobre o seu tamanho numérico. A proposta inicial de Fehring descrevia um quantitativo mínimo de $25^{(1)}$ para, em seguida, determinar um mínimo de $50^{(10)}$, experts como adequado, embora não exista nenhuma justificativa para a apresentação de tais números.

Na prática, tem se mostrado quase inviável nas pesquisas que envolvem validação garantir que os 50 sujeitos encontrados, convidados e que tenham analisado o material enviado (devolvendo-o em tempo hábil) sejam, de fato, experts. Não é incomum que pesquisadores relatem em seus estudos um número inferior ao recomendado ${ }^{(2)}$. Parte disso se deve, provavelmente, ao fato de que pessoas com a expertise necessária estão, em geral, comprometidas com muitos outros compromissos, uma vez que este é um universo limitado por indicadores de qualidade acadêmica e assistencial.

Para atender ao número de avaliadores considerado adequado nos modelos adotados, tenta-se incluir pessoas que apresentam os critérios mínimos recomendados, mas de forma não consistente, pois demonstram pouca experiência clínica ou mesmo são recém pós-graduados. Estes novos pesquisadores, em geral, necessitam ainda desenvolver certas habilidades na condução de estudos e/ou na análise crítica de situações envolvendo diagnósticos específicos em populações específicas. Estas características são desenvolvidas com o tempo e, embora estes sujeitos possuam algumas habilidades, certamente lhes faltam outras.

Além das dificuldades inerentes à obtenção quantitativa e qualitativa de experts, a proposta de Fehring $^{(3)}$ se baseia no cálculo de um índice de validade de conteúdo. Este índice é, na verdade, uma média ponderada que atribui um peso linearmente mais forte quando o expert acredita na adequação de um componente diagnóstico. Em outras palavras, o IVC proposto pelo autor superestima o valor de cada item avaliado; isto é, um item que seria excluído na etapa anterior tem uma chance maior de permanecer e ser avaliado na etapa subsequente. Alguns estudos recentes, utilizando uma abordagem de validação clínica diferente da proposta por Fehring, têm demonstrado que indicadores que apresentam IVC mais baixos, não apresentam bom desempenho após serem avaliados com aplicação de testes estatísticos específicos ${ }^{(6)}$.

Por fim, a etapa de validação clínica tradicionalmente segue a abordagem retrospectiva, ou seja, um pesquisador (expert) avalia um conjunto de pessoas e seleciona aqueles que apresentam o diagnóstico em questão. Em seguida, dois enfermeiros avaliam simultaneamente cada paciente tentando identificar os indicadores clínicos e fatores etiológicos presentes.

Novamente dificuldades e críticas são apontadas neste processo. Para um pesquisador formular com relativa acurácia um diagnóstico, será necessário que o mesmo perceba 
o comprometimento do sujeito. Isto é denominado viés de espectro e significa que os componentes validados são característicos do grau de comprometimento calibrado pelo próprio pesquisador. Também não pode ser omitido o problema representado pelo caráter retrospectivo do método, ou seja, o desfecho (diagnóstico) já é conhecido e a dupla de examinadores tentará identificar o que pode caracterizá-lo ou o que pode tê-lo causado. Desenhos retrospectivos são classicamente reconhecidos pelo viés de seleção(11), ou seja, o conhecimento prévio do desfecho pode levar o avaliador a buscar dados específicos de forma tendenciosa, ou a exagerar em suas conclusões como forma de confirmar uma suspeita. Este problema é particularmente grave na análise de fatores etiológicos na qual o risco de se trocar a relação causa-efeito por efeito-causa aumenta ${ }^{(12)}$.

Para indicadores clínicos a situação não é muito diferente. Se um pesquisador formulou um diagnóstico, isto significa que ele usou um processo de raciocínio clínico no qual identificou indicadores clínicos, agrupou-os e estabeleceu a presença / ausência do mesmo. Logo em seguida, os examinadores vão identificar os (mesmos!) indicadores clínicos e verificar se eles são mais ou menos importantes para aquele diagnóstico inicialmente elaborado. Isto é denominado viés de incorporação e é tido como uma tautologia no processo diagnóstico ${ }^{(13)}$.

A limitação do método de validação clínica também inclui o fato de apenas indivíduos classificados como portadores do diagnóstico ser avaliados quanto à presença de indicadores clínicos e fatores etiológicos. Nesta situação, não é possível verificar a especificidade destes elementos, ou seja, não há como garantir que estes mesmos elementos não estariam presentes entre indivíduos sem o diagnóstico.

Além dos aspectos já mencionados, os quais levam à critica dos modelos tradicionais de validação, a análise da validade dos elementos para o diagnóstico em questão é baseada num coeficiente de confiabilidade que nada mais é do que a frequência média de um indicador clínico identificado pelos dois avaliadores, ponderada pela concordância absoluta entre eles. De forma intuitiva, isto quer dizer que o valor de um indicado clínico é fortemente influenciado por sua frequência. Em epidemiologia isto é conhecido como viés de prevalência $^{(12)}$.

O modelo de diferenciação diagnóstica segue um padrão similar ao modelo de validação clínica, inclusive utilizando o mesmo coeficiente de confiabilidade ${ }^{(3)}$. Embora, os modelos propostos por Fehring apresentem essas limitações, sua importância para a pesquisa sobre validação de diagnósticos é incontestável. Vários estudos foram desenvolvidos com base nestes modelos e ainda hoje muitos os utilizam.

Face às dificuldades e questionamentos apresentados, a busca de outras abordagens que permitam um novo olhar sobre os processos de validação de diagnósticos se faz necessária. Métodos que auxiliem a superar as limitações descritas podem permitir o aperfeiçoamento das classificações de diagnósticos de enfermagem, minimizando as inconsistências e maximizando a capacidade de os enfermeiros identificarem corretamente o fenômeno de enfermagem expresso por um indivíduo. Assim, este artigo descreve a seguir alguns métodos alternativos que recentemente vêm sendo desenvolvidos e utilizados na pesquisa de validação de diagnósticos de enfermagem.

\section{ALTERNATIVAS AOS MÉTODOS CLÁSSICOS DE ANÁLISE DE CONCEITOS}

Um diagnóstico de enfermagem não é um conceito em si, entretanto, cada diagnóstico é composto por um núcleo conceitual. Este núcleo permite o estabelecimento de relações temporais entre fatores que supostamente antecedem e se relacionam para a formulação de um conceito e as consequências de tal formulação. As propostas usuais de análise de conceito denominam estes dois elementos como antecedentes e consequentes ${ }^{(14)}$. Ademais, um conjunto mínimo de características que delimitam o conceito em si constituem o que se denomina de atributos essenciais ${ }^{(14)}$.

Como discutido anteriormente, uma das dificuldades do uso de modelos clássicos da análise de conceito em processos de validação de diagnósticos de enfermagem é justamente a forma como estes elementos são relacionados e analisados. Como o núcleo conceitual está associado ao foco da prática profissional (por exemplo, Autoestima) e não apresenta um elemento que defina um julgamento (por exemplo, Baixa), a análise de conceito mostrará como elementos antecedentes todos os fatores que interferem e ajudam a formar a Autoestima de um indivíduo. Estes elementos incorporam fatores biológicos, sociais e espirituais de uma forma geral. Assim, a questão respondida neste tipo de abordagem seria: "Que elementos influenciam o desenvolvimento da autoestima de uma pessoa?" Se o foco do pesquisador estiver relacionado à revisão do diagnóstico Baixa autoestima situacional, uma parte significativa dos achados serão descartados ou induzirão a inclusão de elementos sem importância clínica.

Se pensarmos em atributos essenciais, o problema se apresenta de forma similar à análise de antecedentes. Estaríamos tentando, neste momento, identificar quais os elementos que caracterizam a autoestima de uma pessoa? Isto é, os atributos seriam os elementos que me possibilitam afirmar que a pessoa tem autoestima. A situação é ainda mais confusa quando é feita a análise de consequentes. Nos modelos clássicos, os consequentes representam situações produzidas a partir da ocorrência do conceito ${ }^{(14)}$. Consequentes do conceito Autoestima poderiam incluir interação social, formação de laços afetivos, produtividade laboral etc. Claramente, estes itens não se assemelham a elementos que compõem um diagnóstico e parecem remeter a metas a serem alcançadas por um indivíduo. Desta forma, embora a análise de conceito seja uma etapa fundamental para a revisão dos elementos que delimitam a profissão, seu uso como etapa de um processo de validação de diagnósticos de enfermagem necessita de reestruturação para permitir um melhor direcionamento a este propósito.

Uma análise de conceito como etapa de um processo de validação de diagnóstico não pode desconsiderar eixos que constituem o diagnóstico como um todo. Assim, embora a busca de material inclua inicialmente o núcleo conceitual, a 
identificação de material relevante para o estudo deverá considerar o elemento julgamento. Ou seja, ao invés de buscar todo o material disponível sobre autoestima, o interesse deve recair sobre a Baixa autoestima. Embora pareça limitante, existem razões para tal atitude.

Num processo de validação de diagnóstico, três elementos são essenciais: a definição, os fatores etiológicos e os indicadores clínicos. Existe uma relação direta entre os antecedentes de um conceito e os fatores etiológicos de um diagnóstico. Todavia, diferentemente da questão de pesquisa usual para antecedentes, neste caso estamos interessados em responder a pergunta: "Que fatores podem levar a uma baixa autoestima entre os indivíduos de uma dada população?"

Retomamos aqui o outro ponto que justifica especificar o foco da análise de conceito: a identificação dos atributos essenciais. Estes elementos devem compor a definição do diagnóstico, ou seja, eles são responsáveis pelo limiar clínico mínimo que permitirá ao enfermeiro decidir pelo estabelecimento do diagnóstico. Um ponto importante neste elemento é que parte da definição deverá incorporar algum(uns) indício(s) clínico(s) observável(eis); ou seja, em muitas situações, indicadores clínicos farão parte da definição do diagnóstico.

Os indicadores clínicos constituem o terceiro elemento de um processo de validação. Em geral, estes são elementos que apresentam maior dubiedade para os pesquisadores. Por um lado, eles podem ser confundidos com antecedentes (fatores etiológicos). Por outro, é sempre difícil estabelecer quais indicadores clínicos são essenciais na determinação de um limiar clínico adequado para estabelecer a presença de um diagnóstico.

A maior dificuldade em utilizar os modelos clássicos de análise de conceitos para estudos de validação de diagnósticos de enfermagem é que nem todos os indicadores clínicos são essenciais para formar a definição de um diagnóstico. Entretanto, todos os indicadores clínicos representam gradientes clínicos de um diagnóstico. Estes gradientes são denominados espectro clínico e representam o nível de comprometimento do diagnóstico apresentado pelo indivíduo.

A identificação de indicadores clínicos é facilitada se atribuirmos a eles um status de consequentes, ou seja, se pensarmos em responder a questão: "Que sinais e sintomas clínicos são apresentados por indivíduos com um diagnóstico em particular?" É obvio que a ocorrência de indicadores clínicos é posterior à instalação de uma resposta humana. A dificuldade em visualizar tal situação talvez esteja associada ao fato de algumas pessoas entenderem o produto diagnóstico como uma situação clínica dicotômica, na qual passamos de um estado a outro de forma abrupta. Claramente, um processo diagnóstico representa uma evolução clínica gradual na qual não temos como estabelecer com total precisão o ponto exato em que uma resposta humana se estabeleceu.

Consequentes definidos a partir dos métodos clássicos de análise de conceito representam metas, caracterizando o diagnóstico como um produto, ou seja, um elemento do processo de enfermagem. Por outro lado, ao utilizar a ideia de indicadores clínicos como consequentes, estabelece-se a noção de diagnóstico como um processo de raciocínio clínico ${ }^{(15)}$ o qual está diretamente associado aos métodos de validação de diagnósticos de enfermagem.

Outro aspecto a ser destacado numa análise de conceito é a captação do material em si. A maioria dos autores descreve a busca em bases de dados, incluindo etapas de revisão integrativa ou mesmo sistemática. A maior limitação destas estratégias é que grande parte dos artigos científicos disponíveis que tratam de pesquisas originais dificilmente abordam fatores etiológicos ou indicadores clínicos. Isto é particularmente verdade para os desenhos que representam alto nível de evidência.

$\mathrm{Na}$ verdade, alguns estudos relatam que a maior parte dos artigos dos quais foram obtidos dados para a análise de conceito eram classificados em níveis de evidência inferiores ${ }^{(6-7,16)}$. Uma possível explicação para este fato é que estudos sobre elementos que compõem um diagnóstico são tratados por estudos observacionais e não por ensaios clínicos. Assim, a utilização de níveis de evidência pode levar a falsa impressão de baixa qualidade da análise de conceito.

$\mathrm{Na}$ verdade, o uso de artigos científicos captados de bases de dados é, em geral, insuficiente para incorporar todos os elementos de um processo de validação ou para construir definições (conceituais e operacionais) para os mesmos. Duas alternativas para contornar esta situação é a incorporação de livros-texto, sobretudo quando o diagnóstico em questão refere-se a aspectos fisiológicos, e a busca de opinião de experts. Esta última estratégia é interessante para a construção de definições quando o material encontrado não apresenta elementos suficientes para tanto.

\section{ALTERNATIVAS AOS MODELOS DE VALIDAÇÃO DE CONTEÚDO}

Sobre as propostas de adequação dos modelos de validação de conteúdo diagnóstico, uma primeira sugestão é a troca do termo validação por análise. Um processo de validação é complexo e não podemos garantir que a opinião de um grupo, geralmente pequeno, de indivíduos possa nos assegurar que um conjunto específico de indicadores clínicos e fatores etiológicos representam o verdadeiro conteúdo de um diagnóstico. Se isto fosse verdade, não seria necessária a etapa de validação clínica de um diagnóstico, pois nossos supostos experts já teriam nos garantido tal validade.

Além disso, se a opinião de experts fosse suficiente, os estudos de validação clínica apenas confirmariam o óbvio e todos os elementos apresentariam estatísticas confirmando tal fato. Entretanto, a grande maioria dos estudos de validação clínica realizados descartou elementos que haviam sido validados na etapa anterior. É claro que uma explicação possível para isto é que, talvez, os experts não possuíam o nível de expertise necessário. Desta forma, esta etapa se aproxima mais de uma pré-análise do que de uma validação propriamente dita, justificando o uso do termo Análise do conteúdo diagnóstico.

Não se pode ignorar também a importância da definição e os clareamentos dos aspectos qualitativos e quantitativos comentados em relação aos experts. Em primeiro lugar é preciso considerar que não existe um consenso na literatura 
internacional sobre o perfil mínimo de experts ${ }^{(2)}$. A proposta de Fehring está baseada em critérios ponderados, mas se apresenta extremamente academicista. Apesar da falta de consenso, muitos autores destacam a importância da experiência clínica na formação de um perfil de expertise, bem como a necessidade de equilíbrio entre experiência e formação acadêmica sólida ${ }^{(7)}$.

Devido à dificuldade de se definir um perfil adequado e de encontrar indivíduos com expertise, alguns estudos descrevem adaptações da proposta de Fehring. Tais adaptações não impediram as críticas usuais aos resultados desta etapa de validação. É provável que grande parte dos sujeitos alocados em estudos de validação apresente um perfil mais próximo de uma proficiência do que de expertise. Assim, termos mais adequados para definir tais sujeitos seriam juízes ou avaliadores.

Muitos estudos referem um número reduzido de experts, justificando ser esta etapa uma abordagem mais qualitativa do que quantitativa. Tal justificativa é prudente se os indivíduos que julgam a pertinência do conteúdo em questão forem, de fato, experts. Todavia, observa-se que tal fato é, na grande maioria das vezes, pouco plausível se analisado sob a ótica da literatura especializada.

Sabe-se que estimativas de tamanhos amostrais são baseadas, entre outras coisas, na precisão e no grau de confiança que se deseja obter do parâmetro em estudo. Por exemplo, Fehring propõe utilizar um índice de validade de conteúdo como parâmetro para aceitação / rejeição de um componente diagnóstico. Neste caso, se partimos de uma suposição de normalidade, considerarmos uma amplitude total deste índice entre 0 e 1, e aceitarmos um erro de estimativa de 0,1 , os cálculos amostrais nos indicariam a necessidade de onze avaliadores. Deve-se ter em mente que algumas suposições podem não ser plausíveis. O IVC proposto por Fehring é um índice tipicamente assimétrico, tornando discutível o pressuposto de normalidade.

Mais recentemente tem se proposto o uso de testes binomiais para comparação de proporções ${ }^{(5)}$. Em tal proposta um item é considerado adequado se um determinado percentual de avaliadores assim o define. Para verificar a aderência à distribuição aplica-se um teste estatístico (conhecido como teste binomial) para verificar se a proporção de avaliadores é ou não estatisticamente igual ou superior ao valor pré-determinado. Desta forma, se os pesquisadores definem como ideal uma proporção de $85 \%$ de aceitação entre os avaliadores e como proporção mínima aceitável um valor de 70\%, o número de avaliadores necessário considerando uma confiança de $95 \%$ será de 22 sujeitos.

\section{ALTERNATIVAS AOS MODELOS DE VALIDAÇÃO CLÍNICA}

Recentemente, algumas alternativas à abordagem clássica do processo de validação clínica de diagnósticos de enfermagem têm sido propostas. Estas alternativas incluem propostas para validação em separado de componentes diagnósticos incluindo a validação de indicadores clínicos e de fatores etiológicos. A validação de indicadores clínicos inclui a análise de acurácia destes indicadores, o estabelecimento de árvores de classificação para a definição de um conjunto mínimo de indicadores, a verificação da ocorrência precoce de indicadores clínicos, e a identificação de um conjunto de indicadores que formam uma variável latente que representaria um diagnóstico. Estas diferentes abordagens mostram aspectos diversos que se destinam a verificar o quão representativo de um diagnóstico podem ser aqueles indicadores clínicos.

Nas abordagens de acurácia de indicadores clínicos adota-se o treinamento de um grupo de enfermeiros para atuarem como diagnosticistas. Utilizam-se definições operacionais para identificar indicadores clínicos, submetendo os achados clínicos ao grupo de diagnosticistas para que os mesmos possam realizar a inferência diagnóstica. Esta inferência é considerada como o padrão de referencia para cálculo das medidas de sensibilidade, especificidade, valores preditivos entre outras ${ }^{(5)}$. Uma das críticas a este modelo é o viés de padrão-ouro imperfeito. Isto significa que a avaliação de enfermeiros treinados e avaliados previamente não representa um procedimento $100 \%$ acurado. Embora isto seja verdade, devem-se levar em conta alguns aspectos: 1) Padrões de referencia perfeitos raramente existem ${ }^{(17)}$; 2) Alguns padrões de referencia tido como perfeitos, na verdade dependem da avaliação de sujeitos treinados. Laudos de biopsias, tomografias ou ressonâncias, por exemplo, são dados por pessoal treinado e sujeito a erros de interpretação(18); 3) Diagnósticos de enfermagem representam respostas humanas que, em sua maioria, não podem ser identificados por dispositivos ou máquinas ${ }^{(5)}$; 4) A literatura internacional recomenda o treinamento e avaliação rigorosa de diagnosticistas nas situações em que estes são responsáveis pela inferência diagnóstica ${ }^{(19)}$.

Ressalta-se que os estudos de acurácia diagnóstica apresentam viés de incorporação, ou seja, os dados a serem calibrados (indicadores clínicos) são usados pelo padrão de referência (diagnosticistas) para determinação do status de cada indivíduo avaliado. Por outro lado, é importante notar que, nesta abordagem, a incorporação afeta todos os indicadores clínicos simultaneamente. Assim, o que ocorre é uma mudança no parâmetro de localização dos estimadores. Em outras palavras, ocorre uma superestimação conjunta de todos os indicadores avaliados, de modo que a comparação entre os mesmos pode ser efetuada, mas os valores das medidas em si devem ser vistos com ponderação. Além disso, o fato de não se estabelecer previamente o status dos indivíduos anula a influência do conhecimento prévio e o viés de seleção.

Árvores de classificação são métodos de análise que se baseiam em etapas metodológicas similares à análise de acurácia. Entretanto, os algoritmos utilizados são diferentes e considerados robustos para a determinação de um conjunto mínimo de indicadores clínicos representativos do diagnósti$\mathrm{CO}^{(20)}$. Eles podem ser úteis na identificação de elementos para compor ou revisar a definição de um diagnóstico.

Modelos de análise de sobrevida representam técnicas baseadas na observação longitudinal de indicadores clínicos e de um diagnóstico relacionado. As etapas metodológicas são similares às duas abordagens anteriores acrescidas do seguimento e mensuração repetida das variáveis. Estes modelos possibilitam verificar quais indicadores clínicos estão 
relacionados à identificação precoce de um diagnóstico, permitindo o estabelecimento de sinais clínicos de alerta para aquele diagnóstico. A análise é baseada em técnicas estatísticas mais avançadas, incluindo equações de estimativa generalizada, modelos de Cox estendido e em paralelo.

Modelos que usam a ideia de variável latente se baseiam na identificação de uma variável oculta (diagnóstico de enfermagem) nos dados, representada por um conjunto subjacente observável (indicadores clínicos). Um dos modelos desta abordagem, que tem sido proposto para aplicação com diagnósticos de enfermagem, é derivado da teoria da resposta ao item e conhecido como modelagem Rasch ${ }^{(13)}$. Esta abordagem considera que a relevância de um indicador clínico depende do quão comum é a ocorrência do indicador (denominado de dificuldade do item) e do espectro do diagnóstico, ou seja, do nível de gravidade do diagnóstico apresentado pelo indivíduo (habilidade da pessoa).

Modelos Rasch são flexíveis, permitindo tratar um diagnóstico de forma dicotômica ou mesmo escalar (dimensional). Esta característica e o fato de não depender de um padrão de referência são as principais vantagens deste método. As limitações do modelo incluem a necessidade de reunir todos os indicadores clínicos para uma estimativa correta dos coeficientes envolvidos, a complexidade do ajuste e interpretação dos modelos, a ocorrência de curvas características discrepantes dificultando a decisão sobre a relevância do item e o fato de suas estimativas se resumirem a uma aproximação da ideia de sensibilidade utilizada em análise de acurácia de indicadores clínicos.

Infelizmente, a interpretação dos coeficientes gerados numa análise Rasch não é direta nem segue um padrão uniforme para outros diagnósticos que apresentem os mesmos indicadores clínicos. Isto impede o desenvolvimento de medidas-resumo para múltiplas populações com um mesmo diagnóstico ou para múltiplos diagnósticos que compartilham um mesmo indicador clínico. Este tipo de comparação é possível nos métodos de acurácia pela aplicação de métodos de metanálise de testes diagnósticos ${ }^{(17)}$.

Um aspecto particular da validação de indicadores clínicos refere-se aos métodos para diferenciação diagnóstica que têm sido propostos. Estes métodos visam estabelecer quais indicadores clínicos auxiliam no estabelecimento de diferenças clínicas entre dois diagnósticos que apresentam certo grau de similaridade. A validação de uma diferenciação diagnóstica deve começar com a análise de conceito. Entretanto, diferente da proposta dos métodos clássicos, o objetivo desta análise deve incluir a avaliação dos conceitos desenvolvidos por avaliadores independentes, a confluência posterior das análises independentes buscando estabelecer os elementos comuns e específicos dos diagnósticos, e a construção das definições operacionais para todos os elementos.

A etapa de análise de conteúdo deve incluir, além da relevância e adequação dos componentes, o grau de representatividade dos elementos para cada diagnóstico. A estrutura desenvolvida nestas etapas, com todos os elementos considerados importantes para os diagnósticos, é utilizada na avaliação de um grupo de sujeitos susceptíveis aos diagnósticos e uma técnica conhecida como análise de correspondências múltiplas pode ser aplicada. Esta técnica permite identificar a confluência de indicadores clínicos para diagnósticos específicos possibilitando definir quais indicadores auxiliam na diferenciação dos diagnósticos.

Apesar de ainda serem escassos os artigos que utilizam os métodos descritos para a validação de indicadores clínicos, estudos que buscam validar fatores etiológicos são ainda mais raros, mesmo se considerarmos os métodos clássicos. Esta escassez leva a um grande problema: a dificuldade de estabelecer causalidade. É sabido que a definição de uma intervenção não pode estar baseada unicamente na resposta humana. Tomemos como exemplo um problema simples e possível de ocorrer tanto em pessoas expostas ao calor quanto ao frio excessivos: a queimadura. É obvio que qualquer tipo de ação preventiva vai depender do fator causador (calor ou frio). Não faz sentido agasalhar alguém que se expõe excessivamente a um calor de $40^{\circ} \mathrm{C}$, nem utilizar bloqueador solar em pessoas expostas a temperaturas muito baixas para prevenir queimaduras.

Assim, o estudo de fatores etiológicos é tão fundamental quanto o de indicadores clínicos. Todavia, a investigação sobre estes elementos requer o uso de métodos específicos. Estudos transversais podem ser utilizados para analisar a razão de prevalência de um diagnóstico entre pessoas expostas e não expostas a fatores etiológicos específicos. Pesquisas recentes propõem a utilização de uma técnica de análise mais robusta denominada regressão Poisson. Esta técnica apresenta valores de razão de prevalência ajustados. Infelizmente, estudos transversais têm baixo poder para estabelecer relações causais, sobretudo, porque representam um retrato momentâneo da situação clínica. Muitas vezes a relação causa-efeito pode ser confundida por uma relação efeito-causa ${ }^{(11)}$.

Dois outros métodos podem ser úteis para a análise de fatores etiológicos: Estudos de caso-controle e estudos de coorte. Estudos de caso-controle se baseiam na comparação de dois grupos definidos a partir da presença ou não de um diagnóstico ${ }^{(12)}$. Um exemplo seria estabelecer como grupo caso indivíduos com hipertensão arterial identificados com Estilo de vida sedentário e como grupo controle pessoas também com hipertensão arterial, porém, sem Estilo de vida sedentário. $\mathrm{O}$ primeiro problema a ser enfrentado pelo pesquisador é o estabelecimento claro dos critérios que utilizará para classificar os sujeitos como caso ou controle. Uma forma de contornar o problema é utilizar indicadores clínicos com melhor acurácia estabelecidos em estudos anteriores para criar tais critérios.

Uma vantagem dos estudos de caso-controle é que eles permitem estudo de múltiplos possíveis fatores etiológicos. Por outro lado, estudos de caso-controle apresentam um alto risco para vieses, tais como os vieses de seleção, memória, revisão e de avaliação ${ }^{(12)}$. Existem procedimentos que ajudam a controlar tais vieses como o cegamento do avaliador, o pareamento e a utilização de técnicas estatísticas específicas para produzir análises ajustadas, tais como, as regressões logísticas ${ }^{(11)}$.

Estudos de coorte representam os estudos observacionais que produzem evidências mais fortes. Coortes permitem calcular taxas de incidência e mensurar o risco de desenvolver determinado diagnóstico entre pessoas expostas a um fato etiológico específico, quando comparadas a pessoas não 
expostas (risco relativo) ${ }^{(12)}$. Diferentemente dos estudos de caso-controle, as coortes são definidas com base na exposição ou não ao fator etiológico. Desta forma, este tipo de estudo permite a análise de um único fator etiológico por vez.

\section{CONSIDERAÇÕES FINAIS}

As abordagens descritas neste artigo são relativamente recentes para validação de diagnósticos de enfermagem e, consequentemente, o material publicado ainda é escasso. Um fator que dificulta a "popularização" destes métodos é a complexidade dos modelos, aliada ao conhecimento incipiente em epidemiologia e bioestatística, aplicado aos processos de validação. Em alguns currículos estas disciplinas são relegadas a segundo plano ou são ministradas ainda baseadas na análise de doenças. Seria interessante se tais disciplinas permitissem aos alunos conhecer modelos de causalidade aplicando-os ao processo de diagnosticar em enfermagem.

\section{REFERÊNCIAS}

1. Fehring R. Methods to validate nursing diagnoses. Heart Lung. 1987;16(6):625-629.

2. Chaves ECL, Carvalho EC, Rossi LA. Validação de diagnósticos de enfermagem: tipos, modelos e componentes validados. Rev Eletrônica Enferm. 2008;10(2):513-520.

3. Garcia TR. Modelos metodológicos para validação de diagnósticos de enfermagem. Acta Paul Enferm 1998;11(3):24-31.

4. Melo RPP, Lopes MVO, Araujo TL, Silva LF, Santos FAAS, Moorhead S. Risk for decreased cardiac output: validation of a proposal for nursing diagnosis. Nurs Crit Care. 2011;16(6):287-294.

5. Lopes MV, Silva VM, Araujo TL. Methods for establishing the accuracy of clinical indicators in predicting nursing diagnoses. Int J Nurs Knowl. 2012;23(3):134-139.

6. Guedes NG. Revisão do diagnóstico de enfermagem estilo de vida sedentário: análise de conceito e validação por especialistas. Fortaleza. Tese [Doutorado em Enfermagem] - Universidade Federal do Ceará; 2011.

7. Benner $\mathrm{P}$, Tanner $\mathrm{C}$, Chesla $\mathrm{C}$. Expertise in nursing practice: caring, clinical judgment, and ethics. 2 th ed. New York: Springer Publishing Company; 2009.

8. Priest HM. Novice and expert perceptions of psychological care and the development of psychological caregiving abilities. Nurs Educ Today. 1999;19(7): 556-563

9. Cavalcante TF. Validação do diagnóstico de enfermagem risco de aspiração em pacientes com acidente vascular cerebral. Fortaleza. Tese [Doutorado em Enfermagem] Universidade Federal do Ceará; 2011.

10. Fehring R. The Fehring Model. In: Carrol-Johnson R, Paquete $M$, editores. Classification of nursing diagnoses: proceedings of the tenth conference of North American
Nursing Diagnosis Association. Philadelphia: Lippincott; 1994. p. 55-62.

11. Hulley SB, Cummings SR, Browner WS, Grady D, Newman TB. Delineando a pesquisa clínica: uma abordagem epidemiológica. 3. ed. Porto Alegre: Artmed; 2008.

12. Rothman KJ, Greenland S, Lash TL. Modern epidemiology. 3. ed. Philadelphia: Lippincott Williams \& Wilkins; 2008.

13. Vargas LCO. Medición en salud: diagnóstico y evaluación de resultados, un manual crítico más allá de lo básico. Columbia: Universidad Industrial de Santander; 2010.

14. Rodgers BL, Knafl KA. Concept development in nursing: Foundations, techniques and applications. Philadelphia: W.B. Saunders; 2000.

15. Lopez M. O processo diagnóstico nas decisões clínicas. Revinter: Rio de Janeiro; 2001.

16. Melo RP. Resultado de enfermagem equilíbrio hídrico no pós-operatório de cirurgia cardíaca: análise do conceito e construção de definições operacionais. Fortaleza. Tese [Doutorado em Enfermagem] - Universidade Federal do Ceará; 2012.

17. Knottnerus A, Buntinx F. The evidence base of clinical diagnosis. Oxford: Blackwell Publishing; 2009.

18. Newman TB, Kohn MA. Evidence-based diagnosis. Cambridge: Cambridge University Press; 2009.

19. Zhou X, Obuchowski NA, McClish DK. Statistical methods in diagnostic medicine. New York: Wiley Interscience; 2002.

20. Chaves DBR. Árvores de Decisão para inferência de Desobstrução ineficaz de vias aéreas e Padrão respiratório ineficaz de Crianças com Infecção Respiratória Aguda. Fortaleza. Tese [Doutorado em Enfermagem] - Universidade Federal do Ceará; 2011. 